\title{
Retroperitoneal teratoma causing ptosis: A case report
}

\author{
LIUYU XU $^{1 *}$, QINGLI ZHAO $^{1 *}$, XUEBEI ZHANG ${ }^{2}$, QING LI $^{1}$ and SHENGLIANG HUANG ${ }^{1}$ \\ ${ }^{1}$ Department of Urology, Shandong Provincial Qianfoshan Hospital, Shandong University; ${ }^{2}$ Department of Nursing, \\ Affiliated Eye Hospital of Shandong University of Traditional Chinese Medicine, Jinan, Shandong, P.R. China
}

Received October 18, 2014; Accepted July 9, 2015

DOI: $10.3892 / \mathrm{ol} .2015 .3573$

\begin{abstract}
The present study reports the case of a patient who had undergone unsuccessful hormone therapy for ocular myasthenia gravis 14-years prior to the current presentation. The diagnosis of ocular myasthenia gravis was once again confirmed by a neostigmine test and repeat nerve stimulation study. Computed tomography scans in an external institution revealed a retroperitoneal cystic tumor with calcification above the left adrenal gland. The tumor was removed via a transperitoneal laparoscopic resection and was diagnosed as a mature cystic teratoma upon pathological examination. A teratoma is a common form of germ cell tumor, but primary teratomas of the retroperitoneum are quite rare in the adult population. Post-operative observation of the patient showed resolution of the ptosis and improved movement of the eyeballs. The potential mechanism was unclear, but the patient's teratoma was mature and may have contained myoid cells with antigenicity for anti-acetylcholine receptor (anti-AchR), as has been established in the thymus. Therefore, the anti-AchR antibody may have been involved.
\end{abstract}

\section{Introduction}

Teratomas are a common type of germ cell tumor. They are composed of well-differentiated or incompletely differentiated elements of at least two of the three germ cell layers (endoderm, mesoderm and ectoderm). Well-differentiated teratomas are termed mature teratomas (1). Teratomas are usually found in the gonadal organs, such as the ovaries and testes (2), while $\sim 15 \%$ of all teratomas are extragonadal (3). The retroperitoneum is the least common location (3) and $26 \%$ of retroperitoneal teratomas are malignant (4). Mature cystic teratomas in the retroperitoneum are most commonly identified in the first 6 months of childhood and in early adulthood (5) with an incidence of only 10-20\% in adults

Correspondence to: Dr Shengliang Huang, Department of Urology, Shandong Provincial Qianfoshan Hospital, Shandong University, 16,766 Jingshi Road, Jinan, Shandong 250014, P.R. China E-mail: hsldoctor@163.com

${ }^{*}$ Contributed equally

Key words: retroperitoneal teratoma, adrenal tumor, ptosis aged $>30$ years old (6). However, many teratoma patients are asymptomatic and thus, are not diagnosed until much later in childhood or adulthood. Furthermore, primary retroperitoneal teratoma in adults is quite rare, accounting for $<10 \%$ of all primary retroperitoneal tumors (5). Teratomas in this location are typically well developed and can occasionally resemble normal fetal elements on computed tomography (CT) scans (7). The symptoms of teratoma vary depending on the tumor location and organ of origin. Patients with ovarian teratomas often present with abdominal or pelvic pain, which is caused by torsion of the ovary or irritation of its ligaments (8). Diagnostic techniques include ultrasound, magnetic resonance imaging and computed tomgraphy. However, definitive diagnosis is based on tumor histology (7). At present, the treatment of choice is complete surgical removal, which exhibits a good prognosis in benign teratomas, however, for malignant tumors, chemotherapy treatment is also administered following surgery (9). A previous study of 183 infants and children diagnosed with teratoma, revealed that the 10-year event free and overall survival rates following surgery were $90.4 \%$ and $98.0 \%$ respectively (10). Furthermore, immature teratomas are associated with a significantly higher mortality rate than mature teratomas (11). Ocular myasthenia gravis is believed to be an autoimmune disease with the postsynaptic defect of neuromuscular transmission as the common feature (12). Neuromuscular transmission failure in myasthenia gravis is most commonly elicited by auto-antibodies to the acetylcholine receptor (AchR) (12). No cases of retroperitoneal teratoma associated with myasthenia gravis have been reported in the literature thus far. The present study reports a case of a primary retroperitoneal mature teratoma in an adult with ocular myasthenia gravis.

\section{Case report}

A 22-year-old male was referred to Shandong Provincial Qianfoshan Hospital (Jinan, China) on September 1, 2014, from an external institution for the treatment of persistent ptosis and limited eyeball movement. A neostigmine test demonstrated that symptoms associated myasthenia gravis improved following injection with $1 \mathrm{mg}$ neostigmine, while repeat nerve stimulation decreased electrical potential of ocular muscles. Thus, the diagnosis of ocular myasthenia gravis was confirmed by neostigmine test and repeat nerve stimulation. The patient had previously been treated unsuccessfully with adrenal cortical hormone (prednisone and urbason) 14 years 


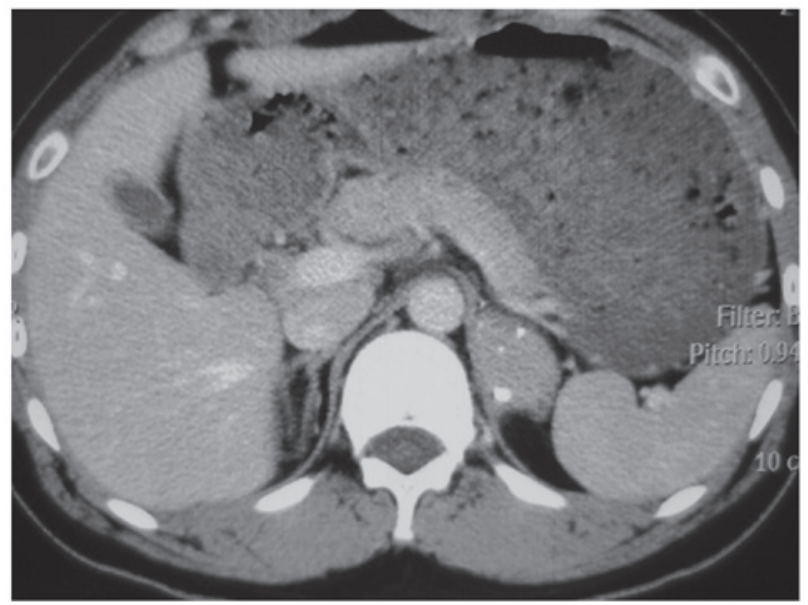

Figure 1. Computed tomography scan showing calcification in the tumor

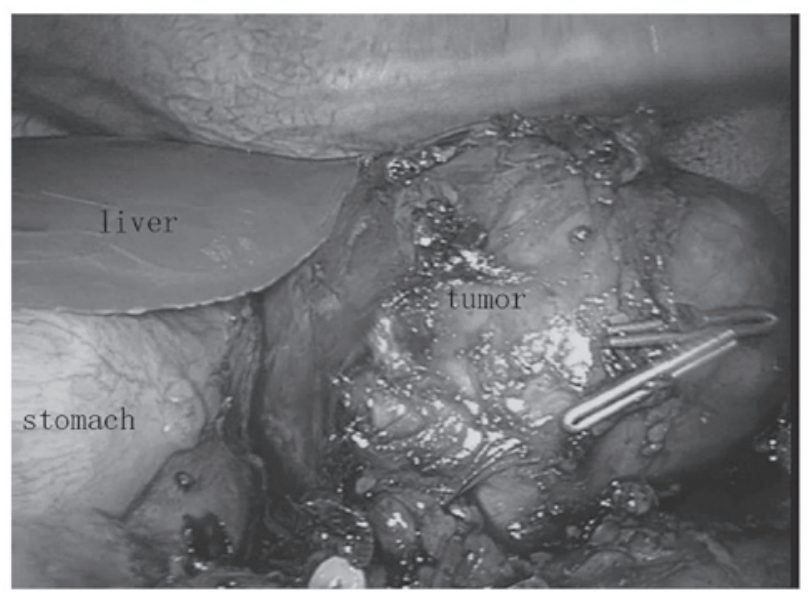

Figure 2. Surgical image showing the tumor featured like a stomach. This was followed by an intraoperative gastroscopy.

prior to the current presentation. A thoracoabdominal CT scan, which was performed to exclude thymoma, revealed a 5.8x2.9-cm ovoid-shaped retroperitoneal tumor with dense, irregular calcification in the soft tissue above the left adrenal gland (Fig. 1). There was no tumor in the thymus and the levels of adrenal cortical hormone in the serum were normal.

The patient was subsequently treated with a transperitoneal laparoscopic resection of the tumor. The tumor was located above the left adrenal gland, left and to the bottom of the stomach, adherent to the diaphragm. During the procedure, the capsule wall was unintentionally cut open and a large quantity of white viscous liquid flowed out. A nodule of $\sim 0.5 \mathrm{~cm}$ in diameter was also found in the upper pole of the left adrenal gland and was resected during the surgery. Histological analysis demonstrated that the retroperitoneal tumor was a mature cystic teratoma and that the adrenal nodule was cortical nodular hyperplasia. The retroperitoneal tumor was a solid cystic lesion with thick regular borders, and well-differentiated components within the cyst, while the cortical nodular hyperplasia identified in the adrenal gland consisted of proliferative and hypertrophic vacuolated cells, containing numerous lipid droplets. On post-operative day 1 , the ptosis had disappeared and the eyeball movement was almost normal. Written informed consent was obtained from the patient for publication of this study.

\section{Discussion}

Teratoma is a common form of germ cell tumor, containing all three germ cell layers. Teratomas are classified as mature or immature, depending on the degree of differentiation of its components (7). The tumors are most commonly observed in the gonads of newborns and children. Primary teratomas of the retroperitoneum are not usual in the adult population (5), and most adult cases are in females. Teratomas in this location tend to be well developed. In the majority of cases, they present as asymptomatic, making the diagnosis at an earlier stage more difficult (13).

In the present case, the patient had been diagnosed with ocular myasthenia gravis for a long period of time and had previously been unsuccessfully treated with pyridostigmine bromide and adrenal cortical hormone. The orbital CT scan in Shandong Provincial Qianfoshan Hospital was normal, and consultations from a number of departments confirmed the diagnosis. No similar cases have previously been reported in the literature.

The retroperitoneal teratoma was identified by chance in this patient and was characterized by benign cystic features with irregular calcifications. A transperitoneal laparoscopic resection of the tumor was performed. During the surgery, the tumor exhibited the same physical appearance and was proximal to the stomach, and as we had not previously encountered this situation, an intraoperative gastroscopy was performed. The tumor was shown not to communicate with the stomach or be a part of it (Fig. 2). A resection was then perfomed, during which the tumor capsule was inadvertently cut. A large amount of white viscous liquid was released and then suctioned, and the whole capsule was eventually removed completely. Unexpectedly, the next day, the ptosis was found to have disappeared and the patient's eyeballs were able to move in almost all directions.

As a classic autoimmune disease, myasthenia gravis is believed to be caused by impairment of the AchRs induced by anti-AchR antibody (12). In the present study, the patient's teratoma was mature and may have contained myoid cells with antigenicity for AchR, as has been established in the thymus (14). The existence of this tumor could have led to the continuous stimulation of anti-AchR antibody and unsatisfactory therapeutic efficacy with hormones. Although it is known that certain lung cancers can cause myasthenia gravis, it was unclear whether there was an endocrine substance secreted by the tumor that led to the ptosis due to a lack experimental tests (15). Long-term follow-up should be performed to determine if the patient's ptosis is resolved and to observe whether any recurrence of the teratoma will occur as a result of the tumor rupture.

The present study reported a rare case of a retroperitoneal teratoma accompanied by ptosis, which was treated successfully by a complete resection of the tumor. The potential mechanism was unclear, but we hypothesized that the anti-AchR antibody may have been involved. The diagnosis of a retroperitoneal teratoma could be made on the basis of imaging studies, and the gold standard treatment strategy for this neoplasm is surgical resection without rupture. 


\section{References}

1. Prokhorova TA, Harkness LM, Frandsen U, Ditzel N, Schrøder HD, Burns JS and Kassem M: Teratoma formation by human embryonic stem cells is site dependent and enhanced by the presence of Matrigel. Stem Cells Dev 18: 47-54, 2009.

2. Shindo K, Ueda J, Toubo T, Nakamura M, Oda Y, Eguchi T and Tanaka M: Primary carcinoid tumor in a retroperitoneal mature teratoma: Report of a case. Surg Today 43: 694-697, 2013.

3. Bedri S, Erfanian K, Schwaitzberg S and Tischler AS: Mature cystic teratoma involving adrenal gland. Endocr Pathol 13: 59-64, 2002.

4. Scott AL, Abbassi-Ghadi N, Archer CM, Swamy R and Gupta S: Neuroendocrine carcinoma arising within a retroperitoneal mature teratoma. Ann R Coll Surg Engl 92: W5-W8, 2010.

5. Gatcombe HG, Assikis V, Kooby D and Johnstone PA: Primary retroperitoneal teratomas: A review of the literature. J Surg Oncol 86: 107-113, 2004

6. Panageas E: General diagnosis case of the day. Primary retroperitoneal teratoma. AJR Am J Roentgenol 156: 1292-1294, 1991.

7. Peterson CM, Buckley C, Holley S and Menias CO: Teratomas: A multimodality review. Curr Probl Diagn Radiol 41: 210-219, 2012.

8. Sundar S, Umman P and Chisthi M: Mature ovarian teratoma presenting as small bowel obstruction. Indian J Surg 75: 411-413, 2013.
9. Göbel U, Schneider DT, Calaminus G, Haas RJ, Schmidt P and Harms D: Germ-cell tumors in childhood and adolescence. GPOH MAKEI and the MAHO study groups. Ann Oncol 11: 263-271, 2000

10. Lo Curto M, D'Angelo P, Cecchetto G, et al: Mature and immature teratomas: Results of the first paediatric Italian study. Pediat Surg Int 23: 315-322, 2007.

11. Yoneda A, Usui N, Taguchi T, Kitano Y, Sago H, Kanamori Y, Nakamura T, Nosaka S and Oba MS: Impact of the histological type on the prognosis of patients with prenatally diagnosed sacrococcygeal teratomas: The results of a nationwide Japanese survey. Pediatr Surg Int 29: 1119-1125, 2013.

12. Blissitt PA: Clinical practice guideline series update. J Neurosci Nurs 45: 317, 2013.

13. Yamasaki T, Yagihashi Y, Shirahase T, Hashimura T and Watanabe C: Primary carcinoid tumour arising in a retroperitoneal mature teratoma in an adult. Int J Urol 11: 912-915, 2004.

14. Keijzers M, Nogales-Gadea G and de Baets M: Clinical and scientific aspects of acetylcholine receptor myasthenia gravis. Curr Opin Neurol 27: 552-557, 2014.

15. Lee JH, Shin HY, Kim SM and Sunwoo IN: A case of lambert-eaton myasthenic syndrome with small-cell lung cancer and transient increase in anti-acetylcholine-receptor-binding antibody titer. J Clin Neurol 8: 305-307, 2012. 